\title{
Genome wide analysis of heat shock transcription factor (HSF) family in chickpea and its comparison with Arabidopsis
}

\author{
Syed Adeel Zafar ${ }^{1,3}$, Muzammil Hussain ${ }^{2}$, Mubashar Raza ${ }^{2}$, Hafiz Ghulam Muhu-Din Ahmed ${ }^{3}$, Iqrar \\ Ahmad Rana ${ }^{4}$, Bushra Sadia ${ }^{4,5}$, Rana Muhammad Atif ${ }^{3,5}$ * \\ ${ }^{1}$ Institute of crop science, Chinese Academy of agricultural sciences, Beijing, China \\ ${ }^{2}$ Institute of Microbiology, Chinese Academy of Sciences, Beijing, China \\ ${ }^{3}$ Department of Plant Breeding and Genetics, University of Agriculture, Faisalabad, Pakistan \\ ${ }^{4}$ Center of Agricultural Biochemistry and Biotechnology, University of Agriculture, Faisalabad, Pakistan \\ ${ }^{5}$ US-Pakistan centre for Advanced Studies in Agriculture and Food Security, University of Agriculture, \\ Faisalabad, Pakistan
}

*Corresponding author: dratif@uaf.edu.pk, adeelzafarpbg@gmail.com

\begin{abstract}
Plants cope with thermo-stress by increased expression of heat shock genes. These genes encode various heat shock proteins (HSPs) which rapidly accumulate and protect plants following hasty heat stress. Heat shock transcription factors (HSFs) primarily regulate expression of $H S P$ genes by deciphering conserved binding motifs in promoter region. We retrieved HSF genes of Arabidopsis and chickpea from the online data bases and analyzed their structure and properties using bioinformatics tools. Here, we reported 20 nonredundant genes encoding HSF domain containing proteins in chickpea. Comparative phylogenetic analysis of $H S F$ genes with Arabidopsis revealed four major groups with several paralogous and orthologous genes. Gene localization studies showed that $H S F$ genes are unevenly distributed across all of the eight chromosomes. Segmental duplications were principally involved in $H S F$ gene family expansion during evolution. HSF genes predominantly contain a single intron. However, quite a few genes also retain two introns, which suggest gain of intron during the evolutionary process. Combined conserved-domain analysis of Arabidopsis and chickpea HSF proteins revealed presence of 19 most common domains. Comparison of conserved domains with phylogenetic tree has shown that some domains were present in a clade-specific manner. The presence of multiple conserved domains in HSF proteins suggested that the respective genes originate from duplication events. Our in-silico work may prove helpful in understanding the evolutionary pathways of HSFs in chickpea.
\end{abstract}

Keywords: Heat stress; Cicer arietinum; In-silico; Phylogenetic analysis; Gene evolution.

Abbreviation: GSDS_gene structure display server; HSP_heat shock proteins; HSFs_heat shock transcription factors; HSBPs_heat shock factor binding proteins; HSE_heat shock elements; Car_Cicer arietinum.

\section{Introduction}

Climate change and global warming has become menace for crop health. Continuous increase in temperature poses significantly negative effect on crop growth and yield (Bita and Gerats, 2013). Plants activate stress responsive pathways to tolerate damaging effects of thermo-stress. Among them, rapid accumulation of heat shock proteins (HSPs) is central. These HSPs are multifunctional like helping in protecting cells against stress damage, folding; intracellular distribution; degradation of proteins, and in signal transduction chains (Hartl et al., 2002; Young et al., 2003). Heat shock proteins are encoded by several heat shock genes and heat shock factor binding proteins (HSBPs) control the expression of these genes (Chen and Zhang, 1997). However, heat shock transcription factors (HSFs) primarily regulate the expression of heat shock genes by recognizing the conserved binding motifs (heat stress element, HSE) which exist in their promoter region. HSEs have an inverted repeat region, which contains a varying number of the DNA sequence (5'nGAAnnTTCnnGAAn-3') (Xiao and Lis, 1988; Amin et al., 1988). HSFs utilize their oligomerization domains to form trimmers and function as sequence-specific trimeric DNA binding proteins. These are the terminal compounds of the signal transduction pathway to activate the expression of the HSP genes (Chen et al., 2006). It has been observed that at least three repeat HSEs are required for transcription activation in vivo when bound by HSF proteins (Drees et al., 1997). Under normal circumstances, the inactive state of a monomeric HSF is maintained by the interaction with the molecular chaperones, such as Hsp70 and Hsp90. In response to heat stress, these chaperone complexes are converted from a transcriptional inactive monomer to an active trimmer through combination of their oligomerization domains. As sequence-specific trimeric DNA binding proteins, the active HSFs are capable of recognizing and combining HSEs in the HSF-inducible gene promoters (Wang et al., 2012). HSEs are formed of repetitive palindromic binding motifs of the 5'AGAAnnTTCT-3' sequence upstream of the TATA box in the HSF-inducible genes (Pelham, 1982; Santoro et al., 1998; Guo et al., 2008; Akerfelt, 2010). In plants, $H S F$ genes were first identified in tomato (Scharf et al., 1990). Afterward, several HSF genes were identified in other crop species including Arabidopsis (Nover et al., 2001; Kotak et al., 
2004), rice (Guo et al., 2008), wheat (Yang et al., 2014) and soybean (Li et al., 2014). HsfA1, HsfA2 and HsfB1 from tomato play key role in heat response by regulating the expression of HSPs and other HSFs (Howarth et al., 1993; Mishra et al., 2002). The HsfA4a identified in wheat was found to be involved in the response to heavy metal stress. Over expression of HsfA4a in rice significantly increased the resistance to heavy metal stress (Shim et al., 2009). Therefore, studies on HSF gene family in chickpea is of prime importance to understand the mechanism of heat tolerance. In the present study, we scanned for and integrated all the non-redundant sets of the chickpea HSF genes, determined their chromosomal locations and gene structure, discovered the conserved binding motifs in their proteins and predicted their protein structures by available software and network stations. These results will help in understanding the evolutionary history and functions of HSFs, and in improving the heat tolerance of chickpea.

\section{Results and Discussion}

\section{Identification of HSF proteins from chickpea genome}

The HSF protein sequences of chickpea and Arabidopsis were obtained from Plant transcription factor database. In initial query, we have obtained 22 chickpea HSFs. However, after finding homology, two of them were removed. Currently, 20 non-redundant HSFs were extracted from the initial 22 HSF sequences of chickpea (Table 1), the polypeptide lengths of chickpea HSFs varied significantly from 156 to 500 amino acids. Previously, 25, 22, 21, 38, and 13 HSFs were identified in rice, Arabidopsis, cucumber, soybean and Triticum urartu with polypeptide length ranged from 249-514, 244-495, 184-560, 213-510, and 266-567 amino acids, respectively (Guo et al., 2008; Zhou et al., 2013; Li et al., 2014; Yang et al., 2014). Isoelectric points (IP) of chickpea HSF proteins were also diverse ranging from 4.42 to 9.25. Formerly, a wide range of IP was also observed in cucumber from 4.70 to 9.10 (Zhou et al., 2013), soybean from 4.35 to 9.92 (Li et al., 2014), T. urartu from 4.59 to 9.0 and Aegilops tauschii from 4.86 to 9.76 (Yang et al., 2014). The molecular weight of chickpea HSF proteins ranged from 55.18 KDa (CarHSF8.3) to 17.77 KDa (CarHSF4.3).

\section{Phylogenetic tree}

In order to analyze the evolutionary relationship among chickpea and Arabidopsis thaliana HSFs, a phylogenetic analysis was done to make a combined phylogenetic tree. The 20 non-redundant chickpea HSFs protein sequences along with 22 Arabidopsis HSFs protein sequences were used to make combined phylogenetic tree (Fig 1). Comparative phylogenetic analysis of chickpea and Arabidopsis HSF transcription factors revealed four major groups of $H S F$ genes with several paralogous as well as orthologous genes. Each group contained both chickpea as well as Arabidopsis HSFs. Previously, the combined phylogenetic analysis of rice and Arabidopsis HSFs divided the phylogenetic tree in three main clusters (Guo et al., 2008). Phylogenetic analysis of cucumber and Arabidopsis HSFs also divided the HSFs in three main clusters (Zhou et al., 2013).

\section{Chromosomal location of chickpea HSFs}

The chromosomal locations of the 20 CarHSF genes were investigated according to genome sequencing data of chickpea. It was revealed that, 19 chickpea $H S F$ genes were unevenly distributed across all of the eight chromosomes, with the exception that one HSF gene (XP_004515711.1) was located in scaffold as its location has not yet been assembled (Fig 2). Evolutionary studies suggested that segmental duplications were principally involved in $H S F$ gene family expansion during evolution. Chromosome 1 and 2 carried only one $H S F$ gene, chromosome 3, 5 and 7 each carried two HSF genes, chromosome 6 carried 3 HSF genes and chromosome 4 and 8 carried four HSF genes. Several reports regarding gene localization studies of various crops reported the presence of $H S F$ genes on chromosomes in a very uneven manner. The identified 22 (Arabidopsis) and 25 (rice) $H S F$ genes were distributed unequally on all the five and twelve chromosomes, respectively (Guo et al., 2008). Similarly, in case of cucumber, $20 \mathrm{CsHSF}$ genes were distributed on all the seven chromosomes but in a very uneven manner. However, the remaining one CsHSF gene was present on scaffold chromosome (Zhou et al., 2013). In case of soybean, the 38 identified $G m H S F$ genes were distributed unevenly on 15 out of 20 chromosomes ( $\mathrm{Li}$ et al., 2014).

Discovery of conserved motifs from chickpea and Arabidopsis HSFs

We have identified 19 conserved motifs from 42 HSF sequences of chickpea (20) and Arabidopsis (22). The number of conserved motifs range between 3 and 9 in each chickpea gene. However, in case of Arabidopsis it ranged from 3 to 10 (Fig 1). CarHSF6.3 had the least number of motifs (motif 1, motif 5 and motif 16), whereas CarHSF6.2 and CarHSF8.3 both have 9 motifs which was the highest number in chickpea genome. Motif 1 was found to be conserved in all CarHSF gene family members. Zhou et al. (2013) reported 15 conserved motifs in 21 cucumber HSF proteins and the number of motifs in each gene varied between 3 and 12. In contrast, five conserved domains were identified in soybean HSFs (Li et al., 2014).

\section{Gene structure}

Gene structure analysis has shown that $H S F$ genes predominantly contain a single intron (Fig 1). However, quite a few genes also retain two introns, which suggest gain of intron during the evolutionary process. It was observed that only three out of 20 chickpea $H S F$ genes i.e., CarHSF8.1, CarHSF4.3 and CarHSF3.2 have two introns. Similarly two out of 22 Arabidopsis HSF genes i.e., AtHSF-01 and AtHSF14 have double introns, others have single intron. Similarly, in previous research, gene structure analysis revealed that soybean $H S F$ genes contain single intron except for one gene which had two introns ( $\mathrm{Li}$ et al., 2014). Similarly, in cucumber HSF genes predominantly contain a single intron while two genes had double introns with only one gene containing three introns (Zhou et al., 2013). In recent years, the studies on the role of introns have gain considerable success. Studies in fungi, nematodes, insects, mammals and plants suggest that introns not only play role in regulation of gene expression, but also involved in gene evolution (Rose, 2008). Interestingly, gene structure including the position of introns/exons and gene size of AtHSFO9 and AtHSF10 is very similar which suggest that these genes may be the result of gene duplication. Furthermore, the position of introns and exons of CarHSF8.4 and AtHSFO3 is very alike, which may suggest their co-evolution in history or it may be the result of horizontal gene flow. 
Table 1. Detailed Information of the CarHSFs including gene ID, gene size (Mb), start and end position on chromosome, protein length (aa), molecular weight (KDa) and Isoelectric point (pI).

\begin{tabular}{|c|c|c|c|c|c|c|c|c|c|c|c|}
\hline Number & Gene name & Gene ID & Chromosome & $\begin{array}{c}\text { Gene Size } \\
(\mathrm{Mb})\end{array}$ & Start & End & Strand +/- & MW (KDa) & $\mathrm{pI}^{\mathrm{g}}$ & Domain $^{\mathrm{h}}$ & Protein size $^{\mathrm{I}}$ (aa) \\
\hline 1 & CarHSF1.1 & XP_004485934.1 & 1 & 48.35 & 3522412 & 3525156 & $(-)$ & 43.7156 & 4.42 & $10-103$ & 382 \\
\hline 2 & CarHSF2.1 & XP_004491224.1 & 2 & 36.63 & 33278060 & 33279460 & $(-)$ & 30.173 & 7.6413 & $26-119$ & 257 \\
\hline 3 & CarHSF3.1 & XP_004493479.1 & 3 & 39.99 & 29262023 & 29264248 & $(+)$ & 27.4287 & 8.1924 & $22-112$ & 239 \\
\hline 4 & CarHSF3.2 & XP_004493725.1 & 3 & 39.99 & 30858979 & 30861997 & $(+)$ & 35.7585 & 7.1178 & $40-131$ & 310 \\
\hline 5 & CarHSF4.1 & XP_004495165.1 & 4 & 49.19 & 1773761 & 1778741 & $(-)$ & 41.5982 & 5.8614 & $41-134$ & 356 \\
\hline 6 & CarHSF4.2 & XP_004496590.1 & 4 & 49.19 & 12653691 & 12655097 & $(+)$ & 30.0481 & 8.2729 & 23-114 & 267 \\
\hline 7 & CarHSF4.3 & XP_004496845.1 & 4 & 49.19 & 13306205 & 13310504 & $(+)$ & 17.7732 & 9.2571 & $33-121$ & 156 \\
\hline 8 & CarHSF4.4 & XP_004497602.1 & 4 & 49.19 & 22123350 & 22126002 & $(+)$ & 50.8657 & 5.0186 & $43-130$ & 446 \\
\hline 9 & CarHSF5.1 & XP_004501463.1 & 5 & 48.17 & 35859064 & 35861334 & $(-)$ & 41.7825 & 4.5677 & $47-139$ & 367 \\
\hline 10 & CarHSF5.2 & XP_004501560.1 & 5 & 48.17 & 36710853 & 36712187 & $(+)$ & 42.5756 & 8.1684 & $24-117$ & 375 \\
\hline 11 & CarHSF6.1 & XP_004503339.1 & 6 & 59.46 & 2717960 & 2720632 & $(-)$ & 41.0083 & 5.3031 & $16-107$ & 359 \\
\hline 12 & CarHSF6.2 & XP_004504369.1 & 6 & 59.46 & 10612315 & 10615415 & $(-)$ & 54.5022 & 5.0939 & $17-110$ & 482 \\
\hline 13 & CarHSF6.3 & XP_004505050.1 & 6 & 59.46 & 16727589 & 16729629 & $(-)$ & 23.642 & 7.7672 & $31-122$ & 202 \\
\hline 14 & CarHSF7.1 & XP_004507947.1 & 7 & 48.96 & 2928586 & 2931061 & $(+)$ & 47.1342 & 5.1723 & $102-194$ & 415 \\
\hline 15 & CarHSF7.2 & XP_004509144.1 & 7 & 48.96 & 12362197 & 12367361 & $(+)$ & 29.7151 & 6.5241 & $8-100$ & 267 \\
\hline 16 & CarHSF8.1 & XP_004511596.1 & 8 & 16.47 & 1870504 & 1872871 & $(+)$ & 37.1964 & 5.1595 & $24-115$ & 337 \\
\hline 17 & CarHSF8.2 & XP_004511933.1 & 8 & 16.47 & 4225628 & 4227497 & $(+)$ & 31.7275 & 7.9589 & $9-100$ & 285 \\
\hline 18 & CarHSF8.3 & XP_004512501.1 & 8 & 16.47 & 8539086 & 8544194 & $(-)$ & 55.1772 & 4.5675 & $32-124$ & 500 \\
\hline 19 & CarHSF8.4 & XP_004512974.1 & 8 & 16.47 & 15082791 & 15084505 & $(+)$ & 37.5416 & 4.7741 & $12-103$ & 332 \\
\hline 20 & XP_004515711.1 & XP_004515711.1 & Unplaced Scaffold & & 449400 & 451382 & $(+)$ & 36.799 & & $29-120$ & 332 \\
\hline
\end{tabular}

Information about gene ID, protein length, MW, pI, chromoson

http://www.ncbi.nlm.nih.gov/gene?cmd=Retrieve\&dopt=full_report\&list_uids=101515265 


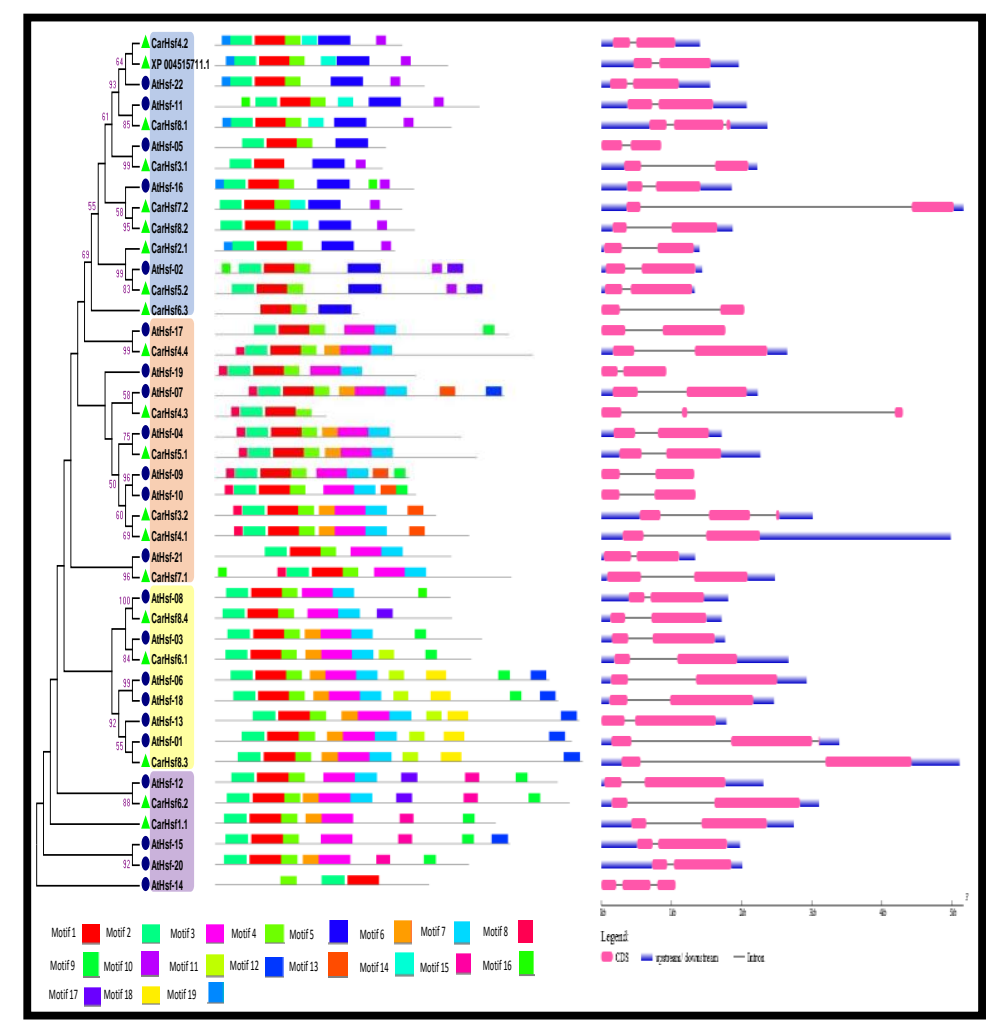

Fig 1. Comparative phylogenetic analysis, conserved domain analysis and gene structure analysis of HSFs of chickpea and Arabidopsis. (A) Phylogenetic tree showed 4 major HSF groups. Green triangles and black circles represent chickpea and Arabidopsis HSFs, respectively. (B) Various conserved domains (colored boxes) identified in Car and At HSFs are shown. (C) Gene structure analysis revealed the presence of single intron in most of the HSF genes of chickpea and Arabidopsis.

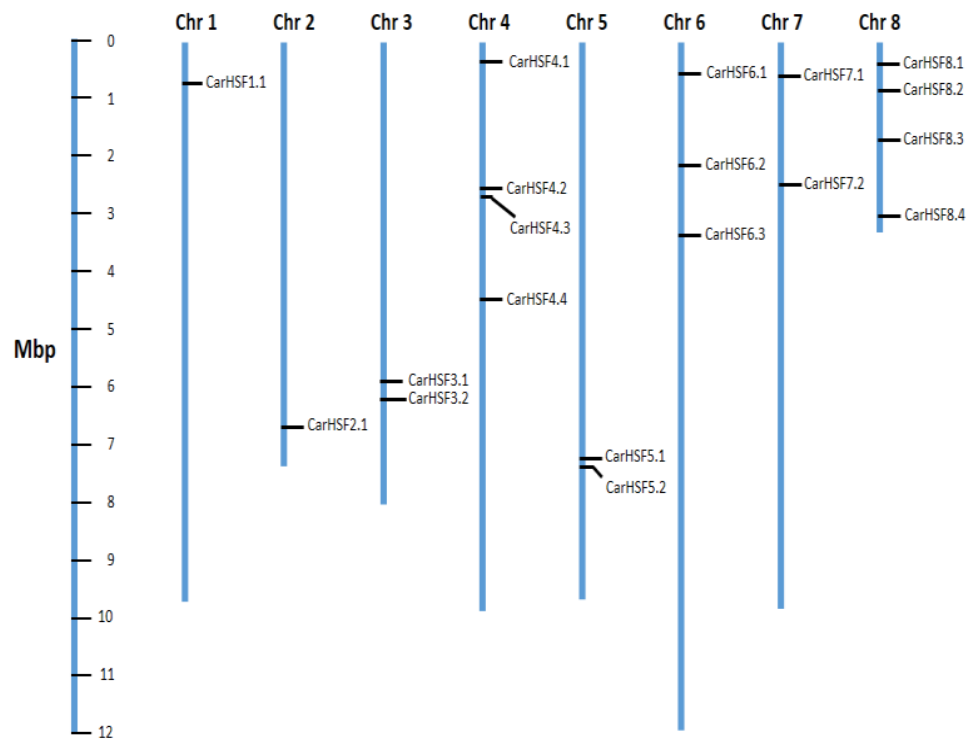

Fig 2. Distribution of Chickpea $H S F$ genes on the chromosomes. The scale bar represents Mega bases $(\mathrm{Mb})$ and name of each chromosome is shown at the top of blue bars. All the HSF genes are unevenly distributed on eight chromosomes. 


\section{Materials and Methods}

\section{Data base search and sequence retrieval}

The whole genome of Cicer arietinum was downloaded from website of International crops research Institute for the SemiArid Tropics (http://www.icrisat.org/). Protein and coding sequences of HSFs of chickpea and Arabidopsis were downloaded from plant transcription factor database v3.0, Center for Bioinformatics, Peking University, China (http://planttfdb.cbi.pku.edu.cn/) while genomic sequences were obtained from National Center for Biotechnology Information (NCBI) database (http://www.ncbi.nlm.nih. gov/). The information about gene name, chromosomal location of the gene, start and end point, amino acid length, protein molecular weight, isoelectric point (PI), and Orthologous of Arabidopsis were also obtained from plant transcription factor database.

\section{Comparative phylogenetic analysis}

Amino acid sequences of all the identified non-redundant chickpea and Arabidopsis HSFs were aligned using Clustal W (Thompson et al., 1994) program of Molecular Evolutionary Genetics Analysis (MEGA version 6.0) software suite. A phylogenetic tree was constructed by using MEGA 6 software (Tamura et al., 2013) with NeighborJoining criteria (Saitou and Nei, 1987) and 1000 bootstrap replicates.

\section{Gene structure analysis}

Gene structure including introns and exons of chickpea and Arabidopsis $H S F$ genes was investigated by using the online Gene Structure Display Server (http://gsds.cbi.pku.edu.cn/) based on genomic and coding sequences (Hu et al., 2014).

\section{Conserved Motifs analysis}

The conserved motifs within chickpea and Arabidopsis HSF proteins were determined by using the MEME online server (Bailey et al., 2015). The parameters were set as follows: maximum numbers of different motifs, 19; minimum motif width, 12; maximum motif width, 52; other parameters retained their default settings.

\section{Chromosomal location of chickpea HSF genes}

The identified non-redundant chickpea $H S F$ genes were mapped on all the eight chickpea chromosomes on the basis of the information obtained from NCBI using MapDraw software (Liu and Meng, 2003). After locating all the genes on different chromosomes, they were assigned new names on the basis of their location on the chromosomes. For example, the gene which was located on the chromosome number 1 at the start was assigned the name as CarHSF1.1 and the gene present on the second chromosome was assigned as CarHSF2.1. Similarly, the first gene on chromosome number 3 was given name as CarHSF3.1 while the next gene on the same chromosome as CarHSF3.2 and so on.

\section{Conclusion}

In this study, 20 non-redundant HSF genes were identified from the sequenced chickpea genome which was distributed across all the eight chromosomes. These chickpea HSF proteins contain 19 conserved motifs. On the basis of phylogenetic analysis, these genes can be classified into four major groups. Gene structure analysis revealed that CarHSF genes predominantly contain a single intron with the exception of three genes which had two introns. This study may provide new insights for the functional characterization of chickpea HSF genes.

\section{Acknowledgements}

We thank the anonymous reviewers for helpful comments to the manuscript.

\section{Reference}

Akerfelt M, Morimoto RI, Sistonen L (2010) Heat shock factors: integrators of cell stress, development and lifespan. Nat Rev Mol Cell Biol. 11: 545-555.

Amin J, Ananthan J, Voellmy R (1988) Key features of heat shock regulatory elements. Mol Cell Biol. 8: 3761-3769.

Bailey TL, Johnson J, Grant CE, Noble WS (2015) The MEME Suite. Nucleic Acids Res. 37: 202-208.

Bita CE, and Gerats T (2013) Plant tolerance to high temperature in a changing environment: scientific fundamentals and production of heat stress-tolerant crops. Front Plant Sci. 4: 273.

Chen JN, and Zhang XT (1997) New progress in research on functions of heat shock protein in human and plants. Hereditas. 19: 45-48.

Chen XJ, Ye CJ, Lu HY (2006) Cloning of GmHSFA1 gene and its overexpression leading to enhancement of heat tolerance in transgenic soybean. Acta Genet Sin. 28: 14111420.

Drees BL, Grotkopp EK, Nelson HC (1997) The GCN4 leucine zipper can functionally substitute for the heat shock transcription factor's trimerization domain. J Mol Biol. 273: 61-74.

Guo J, Wu J, Ji Q, Wang C, Luo L, Yuan Y, Wang Y, Wang J (2008) Genome-wide analysis of heat shock transcription factor families in rice and Arabidopsis. J Genet Genomics. 35: 105-118.

Guo L, Chen S, Liu K, Liu Y, Ni L, Zhang K, Zhang L (2008) Isolation of heat shock factor HsfAla-binding sites in vivo revealed variations of heat shock elements in Arabidopsis thaliana. Plant Cell Physiol. 49: 1306-1315.

Hartl, FU, and Hayer-Hartl M (2002) Molecular chaperones in the cytosol: from nascent chain to folded protein. Science. 295: 1852-1858.

Howarth CJ, and Ougham HJ (1993 Gene expression under temperature stress. New Phytol. 125: 1-2.

Hu B, Jin J, Guo AY, Zhang H, Luo J, Gao G (2014) GSDS 2.0: an upgraded gene feature visualization server. Bioinformatics. 31(8): 1296-1297.

Kotak S, Port M, Ganguli A, Bicker F, von Koskull-Doring P (2004) Characterization of C-terminal domains of Arabidopsis heat stress transcription factors (Hsfs) and identification of a new signature combination of plant class A Hsfs with AHA and NES motifs essential for activator function and intracellular localization. Plant J. 39: 98-112.

Li PS, Yu TF, He GH, Chen M, Zhou YB, Chai SC, Xu ZS, Ma YZ (2014) Genome-wide analysis of the Hsf family in soybean and functional identification of GmHsf-34 involvement in drought and heat stresses. BMC Genomics. 15(1): 1009.

Liu RH, and Meng, JL (2003) MapDraw: a microsoft excel macro for drawing genetic linkage maps based on given genetic linkage data. Hereditas. 25: 317-321. 
Mishra S K, Tripp J, Winkelhaus S, Tschiersch B, Theres K, Nover L, Scharf KD (2002) In the complex family of heat stress transcription factors, HsfA1 has a unique role as master regulator of thermo-tolerance in tomato. Gene Dev. 16: $1555-1567$.

Nover L, Bharti K, Doring P, Mishra SK, Ganguli A, Scharf, KD (2001) Arabidopsis and the heat stress transcription factor world: how many heat stress transcription factors do we need? Cell Stress Chaperones. 6: 177-189.

Pelham HR (1982) A regulatory upstream promoter element in the Drosophila hsp 70 heat-shock gene. Cell. 30: 517528.

Rose A. (2008) Intron-mediated regulation of gene expression, nuclear pre-mRNA processing in plants, Springer. pp. 277-290.

Saitou N, and Nei M (1987) The neighbor-joining method: a new method for reconstructing phylogenetic trees. Mol Biol Evol. 4(4): 406-425.

Santoro N, Johansson N, Thiele DJ. (1998) Heat shock element architecture is an important determinant in the temperature and transactivation domain requirements for heat shock transcription factor. Mol Cell Biol. 18: 63406352.

Scharf KD, Rose S, Zott W, Schoffl F, Nover L. (1990) Three tomato genes code for heat stress transcription factors with a region of remarkable homology to the DNAbinding domain of the yeast Hsf. EMBO J. 9: 4495-4501.

Shim D, Hwang JU, Lee J, Lee S, Choi Y, An G, Martinoia E, Lee Y (2009) Orthologs of the class A4 heat shock transcription factor HsfA4a confer cadmium tolerance in wheat and rice. Plant Cell. 21: 4031-4043.
Tamura K, Stecher, G, Peterson, D, Filipski, A, Kumar S (2013) MEGA6: molecular evolutionary genetics analysis version 6.0. Mol Biol Evol. 30(12): 2725-2729.

Thompson JD, Higgins DG, Gibson TJ (1994) CLUSTAL $\mathrm{W}$ : improving the sensitivity of progressive multiple sequence alignment through sequence weighting, positionspecific gap penalties and weight matrix choice. Nucleic Acids Res. 22(22): 4673-4680.

Wang F, Dong Q, Jiang H, Zhu S, Chen B and Xiang Y (2012) Genome-wide analysis of the heat shock transcription factors in Populus trichocarpa and Medicago truncatula. Mol Biol Rep. 39: 1877-1886

Xiao H, and Lis, JT (1988) Germ line transformation used to define key features of heat-shock response elements. Science. 239: 1139-1142.

Yang W, Li J, Liu D, Sun J, He L, Zhang A (2014) Genomewide analysis of the heat shock transcription factor family in 'Triticum urartu' and 'Aegilops tauschii'. Plant Omics. 7(5): 291

Young JC, Barral JM, Ulrich HF (2003) More than folding: localized functions of cytosolic chaperones. Trends Biochem Sci. 28: 541-547.

Zhou S, Zhang P, Jing Z, Shi J (2013) Genome-wide identification and analysis of heat shock transcription factor family in cucumber ('Cucumis sativus' L.). Plant Omics. 6: 449. 